Para enlazar con este artículo / To link to this article:

http://dx.doi.org/10.14198/fem.2019.34.03

Para citar este artículo / To cite this article:

Soria Berrocosa, Soledad. «Invisibles, oubliées, émancipées: Les femmes journalistes françaises avant l'heure». En Feminismo/s, 34 (diciembre 2019): 53-77. Dosier monográfico: Estado actual de la investigación en Literatura francesa y Género: balance y nuevas perspectivas / État présent de la recherche en Littérature française et Genre: bilan et nouvelles perspectives, coord. Angeles Sirvent Ramos, DOI: 10.14198/fem.2019.34.03

\title{
INVISIBLES, OUBLIÉES, ÉMANCIPÉES: LES FEMMES JOURNALISTES FRANÇAISES AVANT L'HEURE ${ }^{1}$
}

\section{INVISIBLE, FORGOTTEN, EMANCIPATED: PRECURSOR WOMEN IN FRENCH JOURNALISM}

\author{
Soledad SORIA BERROCOSA \\ Universidad de Alicante, Alicante \\ ms.soria@ua.es \\ https://orcid.org/0000-0002-5888-7893
}

\section{Résumé}

Lobjectif de cet article est d'aller à la rencontre d'un journalisme féminin français invisible et oublié par l'Histoire du journalisme, ainsi que de mettre en valeur ces femmes pour qui le journalisme fut une jonction avec la littérature ou un moyen de revendication et d'émancipation.

À cette fin, et à partir de nombreux travaux qui existent depuis quelques années, nous tracerons diachroniquement l'évolution de ce journalisme féminin avant l'heure. Ainsi, une approche constructiviste, quantitative et descriptive, nous éclairera sur l'origine de leur marginalisation dans cette profession très masculinisée, ainsi que sur leurs travaux et la réception de ceux-ci.

Ce parcours nous permettra donc de nous rendre compte que, s'il existe bien une Histoire du journalisme masculin, il existe aussi une Histoire du journalisme féminin en France, qui est malheureusement encore peu connue.

1. Le présent article s'inscrit dans le cadre d'aides octroyées par le Ministerio de Educación, Cultura y Deporte (MDECD) pour la Formation du Professorat Universitaire - Ref. 03673/2016.

Los contenidos de la revista se publican bajo una licencia de Creative Commons Reconocimiento 4.0 Internacional (CC BY 4.0)

Feminismo/s 34, diciembre 2019, pp. 53-77 
Mots-clé: femmes journalistes; XVII ${ }^{e}$ siècle; XVIII ${ }^{e}$ siècle; genre; féminisme avant la lettre.

\begin{abstract}
The objective of this article is to seek out an invisible French women's journalism that has been forgotten by the History of Journalism, as well as to highlight those women for whom journalism was a junction with literature or a means of claiming and emancipation.

To this end and based on many studies that have been published in recent years, we will diachronically trace the evolution of this women's journalism ahead of its time. Thus, a constructivist approach, both quantitative and descriptive, will shed light on the origin of their marginalization in this very masculinized profession, as well as on their work and the reception of it.

This journey will therefore allow us to realize that, if there is indeed a History of male journalism, there is also a History of female journalism in France, which is unfortunately still not well known.
\end{abstract}

Keywords: Journalism; Women journalists; Seventeenth century; Eighteenth century; Gender; Feminism avant la lettre.

\title{
1. INTRODUCTION
}

S’il est vrai que, de nos jours, les statistiques ${ }^{(2)}$ reflètent une presque parité entre le nombre de femmes et d'hommes qui pratiquent le journalisme en France, il n'en fut pas de même à ses aurores. En effet, au début, «elles étaient si peu nombreuses» à faire un métier d'hommes, qu'elles n'ont pas été prises en compte lorsque l'histoire du journalisme fut tracée. Mais ce n'est pas une raison pour les laisser en marge, car leurs textes «auraient pu former un véhicule pour la conscience féministe» (van Dijk 4-5).

Cette prise de conscience a donné le jour, depuis quelques années, à différentes analyses sur la place et le rôle des femmes dans la presse écrite française d'hier (Patouillet; Sullerot La Presse féminine; Sullerot Histoire de la presse féminine; Adler; Veauvy et Laura Pisano; van Dijk; Roberts; Pinson;

2. Statistiques d'après une étude réalisée par la Commission de la Carte d'Identité des Journalistes Professionnels (CCIJP), en 2018. [En ligne], consulté le 30 mai 2019. URL: http://www.ccijp.net/article-148-statistiques-ccijp.html.

Feminismo/s 34, diciembre 2019, pp. 53-77 
Lévêque; Planté et Thérenty; Andrin). En effet, il y a encore beaucoup de femmes journalistes du passé ou femmes journalistes avant l'heure qui sont toujours invisibles, méconnues ou simplement ignorées. Leurs travaux le sont également, ainsi que leurs répercussions, autant dans le domaine politique ou social que pour l'émancipation de leur sexe, et ce déjà à partir du XVII siècle, lorsque la presse initie sa trajectoire.

Dans le journalisme primitif, elles ont participé de différentes manières. Beaucoup d'entre elles ont été nouvellistes, libellistes, pamphlétaires, gazetières, épistolières, chroniqueuses, mémorialistes, etc. Certaines ont été brillantes, d'autres beaucoup moins. Mais, cela n'a rien à voir avec leur sexe puisque du côté des hommes, le brio a également connu différents niveaux. Elles ont aussi bien traité des thèmes politiques que littéraires, sociaux, artistiques, événementiels ou autres, et apporté aux différents supports de l'information une vision féminine sui generis.

Pourquoi cette profession a-t-elle été masculinisée? Qui étaient ces femmes journalistes? Quelle place occupaient-elles dans ce milieu? Faisaientelles le même travail que leurs confrères? Comment étaient-elles perçues et considérées? C'est à ces questions que cet article, qui ne peut être qu'un échantillon limité vu l'ampleur du thème, prétend répondre.

En retraçant, en partie, leur itinéraire journalistique par le biais des recherches les plus significatives effectuées depuis les années 80, nous désirons non seulement visibiliser leurs noms et leur travail, mais aussi analyser leur rôle dans la structure hiérarchique de cette profession dans laquelle elles ont fait leur entrée, tout d'abord en «marchant sur des œufs» puis «d'un pas lourd». Car si, aujourd'hui, nous pouvons dire que le journalisme s'est féminisé, il fut un temps où ce n'était pas le cas. Au contraire, ces femmes étaient voilées ou perçues comme des marginales aux yeux de la société, ou encore pire, des imitatrices de leurs pairs masculins ou carrément des conspiratrices.

\section{2. «JOURNALISTE»: UN SUBSTANTIF QUI FAIT DE SON GENRE?}

Selon toute vraisemblance, l'attestation du mot «journaliste» remonte à 1684, sous la plume de Pierre Bayle, dans l'avant-propos de son Dictionnaire Historique et Critique (1647-1706) (Feyel 6). Mais, ce n'est qu'au début du $\mathrm{XVIII}^{\mathrm{e}}$ siècle qu'il apparaît dans un dictionnaire avec son acception. De telle 
manière, nous trouvons dans le Dictionnaire Universel d'Antoine Furetière (1701) une des premières définitions: «JOURNALISTE s. m. Celui qui fait un journal. Il n'y a guère d'écrits mieux reçus du public, ni lus avec plus d'avidité, que ceux des Journalistes» [c'est moi qui souligne].

Évidemment, ce terme ne désignait pas encore la profession telle que nous la connaissons aujourd'hui, mais soulignons, sans pour autant nous scandaliser, étant donné l'époque, que le pronom démonstratif «celui», employé dans la définition, est au masculin. Pourtant, morphologiquement, ce mot isole l'un ou l'autre genre pour le rendre, en quelque sorte, hermaphrodite. D'entrée, ces deux éléments linguistiques confèrent au terme une possession genrée et sexuée à sens unique laissant le féminin et les femmes en dehors. La neutralité est bannie, et le stéréotype créé est prêt à prendre racine dans les mentalités. Le journalisme, à partir de ce moment, est un métier dont les hommes se sont attribué la propriété, et aussi la pratique au cours de l'histoire.

Plus tard, dans la version revue de 1771, ce même dictionnaire complétait la définition comme ceci: «JOURNALISTE s. m. Celui qui fait un journal. On le dit de tous les Auteurs de Journaux, quoiqu'ils n'en portent point ce titre. Il n'y a guère d'écrits mieux reçus du public, ni lus avec plus d'avidité, que ceux des Journalistes. Les Journalistes de Leipsik, les Journalistes de Trévoux. Un Journaliste de Paris, de Hollande, \&c. C'est-à-dire, un de ceux qui travaillent aux Journaux de Paris, de Hollande, \&c.» (257) [c'est moi qui souligne]. Une nouvelle fois, le masculin l'emporte sur le féminin -plusieurs travaux critiques féministes ont questionné cette règle grammaticale (Houdebine; Baudino; Elmiger; Viennot; Kahznadar)-, et nous dirons même plus: le masculin ignore, encore, complètement le sexe opposé lors de sa définition, ainsi que lors de sa détermination lexicale, laquelle insiste sur la phallocratie du genre. Plusieurs travaux critiques féministes ont questionné cette règle grammaticale où le masculin efface le féminin () Les différents dictionnaires suivront leur parcours misogyne (Houdebine, Baudino, Elmiger, Viennot, Kahznadar).

En effet, le XIX $\mathrm{X}^{\mathrm{e}}$ siècle persista dans la même voie, comme le prouve la définition du Dictionnaire de l'Académie Française: «JOURNALISTE. s. m. Celui qui fait, qui rédige un journal, qui travaille, comme rédacteur, à un journal. La profession de journaliste. Il s'est fait journaliste. Le journaliste a été mal informé. Le journaliste anglais prétend que...» (1835, 6édition 2: 
78) [c'est moi qui souligne]. Aussi bien les démonstratifs, comme les pronoms ou les articles définis insistent encore uniquement sur la marque du masculin.

Il en sera de même au $\mathrm{XX}^{\mathrm{e}}$ siècle où, dans le même dictionnaire, la définition apparaît comme suit: «Journaliste $\boldsymbol{n}$. $\boldsymbol{m}$. Personne qui a pour métier de participer à l'élaboration des journaux et autres moyens d'information. Un journaliste politique, parlementaire, littéraire, qui rend compte de l'actualité en ces domaines. Journaliste d'agence. Journaliste sportif. Journaliste de radio,

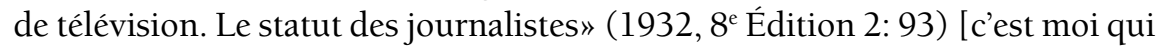
souligne]. Nous remarquons que le genre grammatical et le genre lexical du nom sont toujours au masculin ( $\boldsymbol{m}$.), et même si au début de la définition le terme «Personne» marque une volonté de neutralité, celle-ci suit pourtant en ne mentionnant que l'article indéfini masculin singulier «un». En définitive, la tentative ne reste qu'un coup de poker, et la domination masculine, pour reprendre le titre de l'œuvre de Bourdieu, règne et oppresse le deuxième sexe.

De la même façon, dans l'acception actuelle de plusieurs dictionnaires ${ }^{(3)}$, nous trouvons toujours en tête le substantif «Personne» marquant cette volonté de neutralité dont nous avons parlé. De plus, nous remarquons que le masculin n'apparaît plus ou qu'il a été remplacé par «invariable». Ceci peut porter à deux réflexions: 1 . Serions-nous sur la bonne voie? 2. Serait-ce une stratégie pour apaiser les féministes linguistes qui mettent en question les fondements d'une langue misogyne? En tout cas, malgré le nombre d'études qui traitent à juste titre la problématique du genre des mots ou des métiers, il y a encore un long chemin à parcourir. En réalité, le rôle des dictionnaires est plus important qu'on ne pourrait le croire puisqu'ils contribuent à l'éducation sociale.

Or, contrairement à «journaliste» où il semble que les femmes n'aient pas leur place, il existe un terme pour nommer les journalistes peu talentueux(ses). Du côté masculin, nous avons «journaleux», et du côté féminin, nous avons «journaleuse». Nous voyons que le féminin est appliqué ici sans

3. Voir définitions dans les dictionnaires en ligne: TLFi: «Journaliste, subst. B. - Usuel. Personne dont le métier est d'écrire dans un ou plusieurs journaux»; Reverso Dictionnaire: «Journaliste $n$. inv. 1. Personne qui informe le public par les journaux, la télévision ou la radio. 2. Personne qui traite de l'information dans un système médiatique [c'est moi qui souligne]. Il en est de même pour le Littré ou le Dictionnaire de l'Académie française, entre autres. Aucun ne tient compte du double genre.

Feminismo/s 34, diciembre 2019, pp. 53-77 
aucun problème. En effet, ce terme féminisé est «plus simple et plus idoine au cas d'une femme qui écrit» («On dit que»4). Cette connotation péjorative du terme appliqué aux deux sexes est un exemple clair que, dans la gamme des injures attribuées aux journalistes médiocres, les femmes ont, contrairement au terme «journaliste», leur place. Ainsi, «journaleuse» est à la femme journaliste médiocre, ce que «bas bleu» est à la femme de lettres soit-elle géniale ou médiocre.

En fait, c'est la définition qui donne au mot cette valeur symbolique qui renvoie à l'idée que le journalisme appartient à la gent masculine. Lorsque les fondations de toute structure, soit-elle architecturale, sociale, linguistique ou autre, s'édifient de cette façon, il s'avère nécessaire d'investir dans leurs rectifications.

\section{FEMMES JOURNALISTES DU XVII ${ }^{\mathrm{E}}$ SIECLE}

Après cette mise au point, et sans plus d'ambages, nous passons à retracer les débuts d'un journalisme féminin. Oui, des femmes journalistes avant l'heure ont bel et bien existées. Ainsi, comme le souligne Marguerite Durand, si «le journalisme masculin prétend avoir un roi dans ses ancêtres sous le nom de Louis XIII - ${ }^{(4)}[\ldots]$ les femmes, pour ne pas être en reste ont [...] découvert une royale consœur [...] en la personne de la Duchesse de Longueville, [et fait] moins connu, c'est qu'une femme écrivit de façon suivie dans les mazarinades [...] Suzanne de Nervèze» (1).

Ceci nous renvoie au XVII ${ }^{\mathrm{e}}$ siècle, où les outils médiatiques sont encore ceux du siècle précédent (plaquettes, placards ${ }^{(5)}$, affiches, nouvelles à la main ou lettres en vers manuscrites ou imprimées), et auxquels vient s'ajouter un nouveau genre, ancêtre du journal: la gazette, inaugurée par Théophraste Renaudot (1586-1653) en 1631, sous le nom de La Gazette de France.

4. Le journaliste royal a composé 142 feuillets sur ses expéditions militaires en Lorraine, depuis le 11 septembre 1633 jusqu'au 14 juin 1642, afin qu'ils soient publiés dans la Gazette de Renaudot (Schmit 346-360).

5. Les plaquettes sont des ouvrages comportant un nombre limité de pages. Ce terme s'emploie pour désigner des publications de haute époque et sont considérées les précurseuses du journal, au même titre que les placards, qui sont des écrits qu'on affichait sur un mur, un panneau, pour donner avis au public (Pierrel).

Feminismo/s 34, diciembre 2019, pp. 53-77 
Il est vrai que le siècle précédent voyait déjà naître des plaquettes écrites «par des femmes ${ }^{(6)}$ ou sous une identité féminine». Pourtant, étant donné que l'auteur continue à soutenir qu'«il est difficile de déterminer l'authenticité de ces prises de paroles; dans bien des cas, des hommes sont vraisemblablement les rédacteurs des textes» (Maira), nous ne nous y attarderons pas.

C'est durant la Fronde, période tumultueuse politiquement, qu'il faut souligner une présence attestée des femmes, bien entendu minoritaires, à côté de celle des hommes tels que Renaudot, Jean Donneau de Visé ${ }^{(7)}$ (16381710), Jean Loret (1600?-1665), etc. qui, eux, ont leur place dans l'histoire du journalisme. Ces femmes, par le biais de leurs écrits, «très significatif[s] d'une certaine mentalité féminine» (Grand-Mesnil 215), ont fait un travail journalistique important dans «cette petite presse» (Hatin, Histoire Politique 196) formée par plus de sept milles libelles baptisés Mazarinades; même si leurs noms se perdent parmi tant de noms masculins.

\subsection{Les Frondeuses anonymes des Halles: vision et voix féminines du peuple}

Du côté de la Fronde féminine populaire, Marie-Noëlle Grand-Mesnil souligne l'importance du «langage et opinions des dames de la Halle» (212). En effet, l'auteur inconnu de la Gazette des Halles (1649) fait intervenir un groupe de Dames anonymes qui ont certainement existé, et qui dialoguent sur les principaux acteurs de la Fronde. Ainsi, il imprime dans ce journal ce que Dame Denise, Dame Barbe, Dame Pacquette, Dame Georgette, Dame Nicole, Dame Perette, Jeanne la Crotée, etc., femmes du peuple, «auraient pu écrire si elles avaient su le faire» (Sgard).

La Gazette des Halles était un pamphlet satirique plus qu'un journal, mais il eut tellement de succès qu'il se convertit en la Gazette de la place Maubert, ou Suitte de la Gazette des Halles, puis Suitte de la Gazette de la place Maubert. Cette dernière «semble avoir été destinée à un public féminin» (Grand-Mesnil

6. Ces femmes sont: la Duchesse de Guise (Catherine de Clèves ou Catherine de Nevers) (1548-1633) qui écrivit deux pamphlets: Regrets de Madame de Nemours sur la mort de Messeigneurs de Guyse ses enfans (1589) ainsi que la Remontrance faicte par Madame de Nemours à Henry de Valloys (1589), et Anne d'Este (1531-1607).

7. Créateur du Mercure Galant (1672-1965).

Feminismo/s 34, diciembre 2019, pp. 53-77 
214). Un de ces pamphlets intitulé Entretien sur les affaires de l'Estat donne le ton «d'une certaine mentalité féminine [dont] l'intérêt se porte non sur les faits, mais sur les personnes les plus en vue de l'actualité» (215).

Ces harangères aux usages et langage graveleux «ont eu, sous tous les règnes, le privilège de haranguer les souverains, de porter leurs bouquets et leurs compliments à toutes les fêtes royales, baptêmes et mariages. Comme elles crient tout, elles ont tout chanté, même le régent!» (Karl 330). Puisqu'elles ne savaient pas écrire, leurs voix provenant de leurs chansons ou de leurs dialogues, dans cette place populaire, sont devenus des matériaux très prisés pour les journaux satiriques de la Fronde.

\subsection{Les Frondeuses de haute lignée et les bourgeoises}

Du côté des savantes de haute lignée, Sophie Vergnes, dans son ouvrage intitulé Les Frondeuses. Une révolte au féminin (1643-1661) (2013), aborde et se centre sur l'action politique menée par un groupe de quinze femmes durant la Fronde sous la régence d'Anne d'Autriche. La clé de cette subversion féminine fut indéniablement possible grâce à cette figure féminine au pouvoir, en attendant la majorité de son fils, Louis XIV. D'autre part, il va sans dire que la Fronde fut une époque propice pour le journalisme de femmes puisqu'elle leur a donné la possibilité «d'investir un espace public [...] qui leur était jusqu'alors interdit» (Lévêque 9).

Parmi ces quinze dames, nous trouvons la Duchesse de Longueville (1625-1707), née Marie d'Orléans-Longueville, ou plus connue sous le nom de Marie de Nemours par son mariage avec Henri II de Savoie, duc de Nemours. Elle était la fille de Henri II d'Orléans-Longueville et de Louise de Bourbon-Condé, Mademoiselle de Soissons. Après avoir perdu sa mère lorsqu'elle avait douze ans, son père épousa en secondes noces Anne-Geneviève de Bourbon-Condé, connue aussi sous le nom de Madame de Longueville, et souvent confondue avec elle.

Lorsqu'elle avait vingt-cinq ans, on lui présenta Jean Loret, «un journaliste [autodidacte] avant l'heure ${ }^{8} »$ qui, une fois entré dans le somptueux hôtel de Soissons, construit jadis pour Catherine de Médicis dans le quartier des

8. Nous faisons allusion au titre de l'article de Louis Regnault, consultable en ligne.

Feminismo/s 34, diciembre 2019, pp. 53-77 
Halles où la jeune duchesse tenait salon, décida de créer une gazette sous le mécénat de celle-ci (Lemarchand 234).

Pour faire face à son projet et combler ses nombreuses dépenses d'imprimerie, Jean Loret reçut également une somme assez importante de Mademoiselle de Scudéry (1607-1701). Elle-même offrait «[...] le témoignage, le reflet de la société du XVII ${ }^{e}$, un peu comme [une] journaliste qui [...] narre les faits divers de notre temps» (Verna 309). Le journaliste ne manqua pas de la remercier dans sa Gazette. Cette information donnée par Eugène Hatin (Histoire Politique 326) démontre bien que les femmes du XVII siècle, de quelque manière que ce fut, sont intervenues dans le journalisme avant l'heure.

Le premier numéro de Loret parut en 1650, tout d'abord, sous le titre de La Gazette Burlesque en vers, car à ses débuts, elle était écrite sous forme de lettres versifiées ${ }^{(9)}$ qui étaient également recopiées et distribuées dans les salons les plus en vogue. D'ailleurs, elles eurent un tel succès qu'elles étaient attendues de tous et de toutes avec beaucoup d'impatience. Elles furent même plagiées, ce qui poussa Loret à les imprimer.

En 1652, la nouvelle version s'intitula La Muze historique en l'honneur de la Duchesse qui, non seulement la finançait mais, fait important, fournissait à Loret ses mémoires journalières, afin qu'elles soient publiées dans ce journal hebdomadaire. Preuve en est cette déclaration qui ouvre le premier numéro de la Muze historique:

Le nom de Gazette, qu'on luy a donné autrefois, n'est point quité par mépris; ce n'est que pour le laisser aux relations qui sont faites en proze, au lieu que, celles dont nous parlons étans en vers, on se doit bien imaginer qu'elles sont débitées par l'une des Muses, et mesmes par celle qui a l'intendance de l'histoire, puisqu'elle nous fournit de mémoires journaliers où toute l'histoire du temps est comprize, de sorte qu'à bon droit la dignité de Muze historique luy est attribuée. (Loret 6)

Lauteur y explique le pourquoi du nouveau titre de cette gazette qui traitait de nombreux sujets tels que: "politique, théâtre, musique, littérature, faits divers

9. Voir le Recueil de quelques Gazettes burlesques dédiées à Mademoiselle de Longueville, par le sieur De Lauret (Loret), 1651, dans lequel on trouve ces lettres manuscrites. Consultable sur https://gallica.bnf.fr.

Feminismo/s 34, diciembre 2019, pp. 53-77 
et potins de la Cour et du Tout-Paris » (Regnault 19). En effet, Mademoiselle de Longueville, telle une journaliste avant la lettre, fournissait tous les événements les plus succulents de la Cour à Loret, qui n'avait qu'à les recopier ou les imprimer. La Muze historique est considérée par beaucoup de chercheurs et chercheuses le premier journal féminin.

Aussi, comme en témoignent les articles de Nicole Aronson et de Sophie Vergnes, la belle-mère de la duchesse de Nemours, Madame de Longueville (1619-1679), prit également une part active dans les Mazarinades. Toutes deux mettent en relief leur condition de femme politique émancipée par obligation, en l'absence de leurs frères et mari. Toutefois, même si l'historien Joseph-François Bourgoing de Villefore (1652-1737) prétendit déjà, dans la Vie de Madame la duchesse de Longueville (1738), qu'elle était l'auteure de plusieurs de ces textes revendicatifs et dénonciateurs, Claude Moreau l'a mis en doute (2: 140). Les différents pamphlets et autres écrits invectifs de la duchesse contre Mazarin sont: Lettre de Madame la Duchesse de Longueville au Roi (1650), Apologie pour Messieurs les Princes... (s.d.), Lettre ouverte au roi, Manifeste de Mme la Duchesse de Longueville, Motifs du traité de Mme de Longueville et de M. de Turenne avec le Roi Catholique, Requeste de Mme de Longueville au Parlement de Rouen (Aronson 141).

Ce qui est certain, c'est que la Duchesse n'est pas une femme ordinaire, pourvue de tous les petits défauts qui sont attribués à l'éternel féminin. Sans aucun doute, avec sa grande capacité de résolution, ses actions et sa plume critique, elle «féminisa l'histoire». Cela est «[un] privilège [qui] est réservé à des êtres d'exception, chez qui le sang abolit le sexe» (Vergnes).

À la même époque, Suzanne de Nervèze ${ }^{(10)}$, une femme de lettres et libelliste prolifique, est auteure d'une quinzaine de textes qui font partie des Mazarinades ${ }^{(11)}$. La plupart de ses pamphlets élogiaient Mazarin, régentes et

10. Après avoir consulté différentes sources, dont la B.N.F., nous n'avons pu identifier ni la date exacte de sa naissance ni celle de son décès. À en croire l'auteur (anonyme) de La Fourberie découverte, ou le Renard attrapé, en 1650 elle était, à un certain moment de sa vie, aussi vieille que Marie de Gournay (1565-1645). Par conséquent, elle vécut, au moins quatre-vingts ans. Néanmoins, «elle recevait encore, en 1663, une pension de quatre cents livres du cardinal Mazarin» (Moreau 1: 331).

11. 1. Le Plus heureux jour de l'année par le retour de Leurs Majestés ...; 2. La Lettre d'une bourgeoise de la paroisse Saint-Eustache; 3. La Monarchie affligée ; 4. Le Rieur de la cour aux bouffons satyriques; 5. Les Souhaits accomplis ; 6. La Lettre d'une religieuse

Feminismo/s 34, diciembre 2019, pp. 53-77 
régnants de son époque. Parmi des hommes, tels que Scarron (1610-1660) ou Cyrano de Bergerac (1619-1655), lesquels n'étaient pas encore connus, elle s'est imposée durant de longues années dans le cercle des Illustres Savantes, et ce, malgré les diffamations projetées sur sa personne. Ce ne sont point ses écrits qui ont été retenus ou commentés par ses contemporains ou même les générations suivantes, mais plutôt sa situation précaire ou son âge mûr. Ainsi, il a été dit que «Suzanne de Nervèze, n'avait pas chez elle un bon dîner, suivant l'expression de la Fourberie découverte, ou le Renard attrapé» écrit en 1650 (Moreau 1: XXXI) ou bien encore, en 1905, on pouvait lire dans le Mercure de France «cette vieille gueuze, Suzanne de Nervèze» (Magne LIV: 512).

Ces vers anonymes de l'Épître burlesque, écrits par un des disciples de Scarron, et repris par Moreau, dans son volume 1 de la Bibliographie des Mazarinades, relèvent le talent de Suzanne de Nervèze, ainsi que son implication dans la presse Lesseline ${ }^{(12)} \mathrm{du} \mathrm{XVII}^{\mathrm{e}}$ siècle:

J'aurois bien dès le jour de Mars,

Donné ces vers fort peu gaillards ;

Mais la divine de Nervèze,

A dame Pallas n'en déplaise,

Savante plus qu'elle cent fois,

A, depuis des jours plus de trois,

Tenu la presse Lesseline

Sur maint ouvrage d'œuvre fine,

Qui doit être présenté

À l'une et l'autre Majesté. (331)

Puis, aurait-elle été si insignifiante, alors que son nom et quelques-unes de ses œuvres apparaissent dans la grande Encyclopédie allemande Grosses vollständiges Universal-Lexicon aller Wissenschafften und Künste, de 1740?

présentée au roi ; 7. La Réception du roi d’Angleterre à Saint-Germain; 8. Le Panégyrique royal ; 9. Lettre de consolation à la reine d'Angleterre sur la mort du roi son mari, et ses dernières paroles ; 10. Lettre de consolation au duc de Ventadour,... ; 11. Le Legs royal,... ; 12. La France triomphante sur tous les États et Empires du monde,... ; 13. Le Te Deum des dames de la cour ; 14. Le Discours panégyrique au duc d'Orléans ; 15. Le Discours héroïque. (Moreau 1: 331).

12. Scarron céda son Privilège à l'imprimeur Alexandre Lesselin le 02 février 1655 (Lachèvre 26). Cette information nous permet de supposer qu'elle vivait toujours cette année-là. Lesselin sortit en 1654 son premier numéro de la Muse héroï-comique.

Feminismo/s 34, diciembre 2019, pp. 53-77 
À ce sujet, nous voulons mettre en amont que, parmi les œuvres citées dans ce dictionnaire, il y en a deux qui ne sont pas reprises dans la notice de Diane Desrosiers-Bonin, publiée en 2005 sur le site SIEFAR. Nous les rajoutons afin de compléter la bibliographie de Suzanne de Nervèze: L'agréable pélerine, sa sainte piété E sa sainte retraite (1643); Les consolations universelles sur les plus ordinaires accidens de la vie avec le départ, l'absence E le retour du malheureux Sylvian (1643) (Zedler). En outre, la date du Raisonnement chrétien sur les vertus cardinales diffère dans les deux sources. En effet, alors que Diane Desrosiers-Bonin indique qu'il est de 1643, le dictionnaire allemand prétend qu'il date de 1644 .

Suzanne de Nervèze ne se cantonna pas seulement d'écrire des libelles politiques. Son œuvre se compose également d'un court roman intitulé La Nouvelle Armide, d'un panégyrique féministe intitulé l'Apologie en faveur des femmes (1642), d'épîtres, d'éloges, de dialogues, etc.

Malgré le peu d'études qu'il y a sur l'œuvre de cette libelliste, celles qui existent sont très intéressantes. Ainsi, le mémoire d'Élizabeth Plante, qui se centre sur l'analyse de La Nouvelle Armide ou encore l'article de DesrosiersBonin sur les «Stratégies textuelles pour légitimer sa prise de parole publique et critique» du Rieur de la cour (1649), rendent compte de la diversification littéraire de Suzanne de Nervèze. Par ailleurs, comme le rappelle Desrosiers dans sa notice, d'autres «informations relatives à Suzanne de Nervèze se trouvent également dans l'anthologie de textes féminins inédits des $\mathrm{XVI}^{\mathrm{e}}$ et $\mathrm{XVII}^{\mathrm{e}}$ siècles réunis par Colette $\mathrm{H}$. Winn sous le titre Protestations et revendications féminines (2002), dans Les Muses guerrières d'Hubert Carrier (1996)». Elle-même a également travaillé sur la correspondance de Suzanne de Nervèze dont les résultats restent encore à paraître.

La Bibliographie des Mazarinades compile de nombreux auteurs et de nombreux textes parus sous presse durant la Fronde. Quant aux femmes, bien que moins nombreuses sur la scène de cette presse tantôt réactionnaire tantôt élogieuse, elles ont cependant participé à ce déversement d'informations de façon active. Cette audace, elles l'ont payée en supportant les opinions et commentaires misogynes de leurs confrères. Instigatrices ou conspiratrices étaient des adjectifs très en vogue pour les qualifier. Les ridiculiser était une constante; au point même, comme nous le savons, d'inspirer Molière lorsqu'il créa ses Précieuses ridicules au siècle suivant. Dans ce même sens, ce

Feminismo/s 34, diciembre 2019, pp. 53-77 
dialogue entre Gabriel Naudé (1576?-1653), bibliothécaire, médecin et écrivain du Cardinal Mazarin, chargé par ce dernier d'indemniser Suzanne de Nervèze pour ses écrits, et Jean Camusat (159 ?-1639), vient renforcer notre affirmation:

Mais certes tu aurois bien dequoy t'estonner davantage, si tu connoissois les Autheurs de la plus grand part des libelles, qui ont paru depuis la veille des Rois; car je te puis asseurer que neuf ou dix personnes, dont la plus sçavante ne pourroit pas decliner son nom, en ont fait plus de trois cens: Il n'est pas mesme jusques aux femmes qui ne s'en meslent, tesmoin la Damoiselle Nerveze, laquelle merite au moins cette loüange, qu'elle a fait plus paroistre de bonté, \& de moralité que d'aigreur, dans plus d'une quinzaine qui sont de son invention; \& je te puis fournir bon garent, que les admirables sentiments d'une Villageoise à Monsieur le Prince, sont la quatre ou cinquième des pièces, qu'une simple servante de Librairie, après avoir bien escuré les pots, \& lavé ses escuelles, nous a données en cette occasion. (Naudé 8-9)

Nervèze est citée directement sur un ton à la fois reconnaissant et sarcastique, alors que toutes les autres sont méprisées. En effet, le verbe «se mêler» prend ici une connotation péjorative, puisque les femmes se «mêlent» d'affaires qui, normalement, sont réservées aux hommes.

Dans la dernière partie du fragment, Charlotte Hénault est citée. Selon Moreau elle «était à la fois la sœur et la servante de Jean Hénault, le libraire» (1: XXXI). Apparemment, elle aurait écrit quatre ou cinq pamphlets, d'après les dires de Naudé, tandis que Moreau en compte six qu'il qualifie de «détestables» ( $1: 23)$. De son texte, les Admirables sentiments d'une fille villageoise envoyée à monsieur le prince de Condé touchant le parti qu'il doit prendre (1648), Moreau reprend les paroles suivantes de Naudé: «c'est la quatrième ou cinquième pièce qu'une simple servante de librairie a donnée après avoir bien escuré ses pots et lavé ses écuelles» (1: 22). Cette remarque reflète bien la pensée misogyne de l'époque. La femme qui, entre la lessive, le ménage et la famille, écrit, ne peut forcément pas être prise au sérieux. Malheureusement, ce stéréotype perdurera encore et encore. Lorsqu'elles sortent de la sphère privée, où elles étaient reléguées, pour passer à l'action dans la sphère publique, les femmes étaient immédiatement vilipendées.

Bien qu'il n'y ait presque aucune ou peu d'informations sur elles, nous savons, grâce aux travaux de Moreau et autres chercheurs et chercheuses, que d'autres femmes ont écrit occasionnellement dans les Mazarinades. Par

Feminismo/s 34, diciembre 2019, pp. 53-77 
exemple, Dame de Monterbault-Bouju ou la reine Christine de Suède (16261689), Marie du Cosso et Élisabeth Salète (Carrier, La Presse de la Fronde 21), Claude Scotte, dite La Martingale ou La Sybille Françoise, une soi-disant «pythonisse» (Rosteguy de L'Ancre 223).

Il est vrai que participer occasionnellement dans la presse n'octroie pas le statut de journaliste. Mais, à cette époque, nous savons que beaucoup de feuilles étaient anonymes ou signées seulement avec des initiales afin d'éviter les représailles. C'était le cas, par exemple, de Charlotte Hénault qui signa ainsi Les Admirables sentiments d'une fille villageoise envoyée à monsieur le prince de Condé touchant le parti qu'il doit prendre (1648). Il est donc fort probable que derrière certains de ces textes se cachent des femmes.

Le métier de la presse se compose d'un vaste champ d'activités, et dans le champ de l'édition, il est intéressant de remarquer que plusieurs femmes étaient à la tête d'importantes imprimeries parisiennes qui publiaient tout ce qui sortait sous la Fronde. Plus étonnant (ou pas), c'est qu'elles sont toutes veuves, et par conséquent elles ont hérité le commerce de feu leur mari. Claude Moreau assure que ces éditrices apportaient leur touche personnelle en changeant ou en ajoutant certains éléments aux textes originaux, comme c'était le cas, par exemple, de la veuve J. Guillemot, qui apparemment était très connue puisque plusieurs feuilles sont sorties de son imprimerie (1: 110).

Nous pensons réellement qu'en allant encore plus loin dans le temps, nous trouverions des femmes journalistes avant l'heure. De plus, ne pourrions-nous pas envisager que les écrits historiques de Madame de La Fayette (1634-1693) soient un croisement entre l'événement journalistique de Cour et le fait littéraire? (Ribard) Ou encore, les nombreuses lettres écrites par des femmes, telle que Madame de Sévigné (1626-1696), ne se rapprochent-elles pas très fort de la chronique politique et littéraire? Néanmoins, c'est ce que pense le chroniqueur André Hallays, qui dit que :

À côté de ces chroniqueurs bénévoles dont Mme de Sévigné est la plus illustre, des nouvellistes de profession exerçaient leur industrie et rédigeaient des feuilles manuscrites que l'on appelait des «nouvelles à la main», des «gazettes secrètes», des «gazetins» ou de «petits manuscrits» [qui comprenaient] des informations sur la politique et le théâtre, sur les modes et les mœurs, des anecdotes, des faits divers et des réclames. (1-2) 
Ces femmes combattantes et révoltées du XVII ${ }^{\mathrm{e}}$ siècle, avec pour seule arme leur plume, à la fois littéraire, féministe et journalistique, ancrées dans un absolutisme patriarcal point favorable à leur émancipation, sont certainement des «projets» d'une presse au féminin. À travers leurs textes, indépendamment de leur statut, elles se sont permis de manifester régulièrement ou occasionnellement leurs pensées, leurs préoccupations ou leurs oppositions durant cette période turbulente que fut la Fronde. Aussi, pour délégitimiser leur entreprise, elles étaient souvent dévalorisées et, par conséquent, écartées de l'histoire de la presse.

\section{LES FEMMES JOURNALISTES DES LUMIÈRES}

Le siècle des Lumières n'est pas seulement le siècle des salons, des clubs, du libertinage, des romans épistolaires, du théâtre renouvelé, de l'Encyclopédie ou de la révolte d'un peuple au ventre creux. C'est aussi un siècle de révolution de l'esprit, qui manquait également de pain. Alimenter l'esprit critique et la curiosité intellectuelle va être un objectif à atteindre coûte que coûte, avec les conséquences tragiques que nous connaissons. Les dogmes religieux, politiques et culturels vont être remis en question, et menaceront les respectives institutions qui feront s'ébranler les édifices de la tradition dans tous les champs, même dans celui de la presse. En effet, de nouveaux genres vont apparaître, en accord avec le temps et les lecteurs et lectrices qui sont de plus en plus nombreuses. Ainsi, le journal remplacera la gazette, les journaux littéraires verront le jour, et les journaux pour femmes vont initier leur parcours.

\subsection{Représentation et présence féminines dans la presse du XVIII ${ }^{\mathrm{e}}$ siècle}

Qui dit «esprit critique» et «curiosité intellectuelle», dit aussi «représentation et présence féminine» dans les «médias» d'alors. En effet, elles sont plus nombreuses en ce siècle, bien que toujours en moindre nombre que leurs homologues masculins, à faire du journalisme, mais aussi à en consommer. Oui, les femmes sont plus nombreuses à être consommatrices de journaux. Cette transformation ne plaira pas à tout le monde, et la presse, ainsi que ceux qui la font, seront très critiqués par les littérateurs conservateurs tels que Voltaire (1694-1778) ou Jean-Jacques Rousseau (1712-1778), lequel prétend 
que les journaux sont des «ouvrages éphémères, sans mérite et sans utilité, dont la lecture, négligée et méprisée par les gens lettrés, ne sert qu'à donner aux femmes et aux sots de la vanité sans instruction, et dont le sort, après avoir brillé le matin sur la toilette, est de mourir le soir dans la garde-robe» (4: 220).

Il est vrai qu'au XVIII ${ }^{\mathrm{e}}$ siècle il y eut une certaine préoccupation pour l'instruction, ou plutôt pour l'éducation des filles puisque le nombre d'écoles pour jeunes filles augmenta à cette époque. Beaucoup étaient des écoles de paroisse où le programme pédagogique se limitait à la lecture, au calcul et au catéchisme, et était laissé aux bons soins des religieuses. Dans la capitale, à ces écoles de paroisse, venaient s'ajouter des écoles payantes où le programme pédagogique ne variait guère pour les filles (LAbbé Allain). Rappelons aussi la création de la Maison royale de Saint-Louis à la fin du XVII ${ }^{e}$ siècle par Madame de Maintenon. Cette école accueillait les «Demoiselles de SaintCyr» qui étaient des jeunes filles de la noblesse ainsi que les illégitimes. Malheureusement, ce projet mourut avec la Révolution et la fin des privilèges monarchiques.

Certains contemporains, tel que Nicolas de Condorcet ${ }^{(13)}$, appuyaient le droit à une éducation égale pour les filles et les garçons, ainsi qu'à une égalité des droits politiques. Pourtant, leur tentative laissa la femme en dehors du projet de la Déclaration des Droits de l'Homme et du Citoyen, car la présence des femmes sur la scène publique a toujours suscité chez les hommes une certaine crainte. Quoi qu'il en soit, certaines d'entre elles résistèrent et entamèrent leur parcours de femmes de lettres et journalistes. Malheureusement, il nous est impossible de les citer toutes in extenso et nous nous contenterons de citer celles qui, à notre avis, nous semblent les plus significatives.

\subsection{Les femmes et le journalisme politique au XVIII ${ }^{\mathrm{e}}$ siècle}

Anne-Marguerite du Noyer (1663-1720), née Anne-Marguerite Petit, est considérée la première femme journaliste politique du XVIII ${ }^{e}$ siècle. Après

13. Condorcet écrivit, en 1790, un essai intitulé L'admission des femmes au droit de cité où il dénonce la violation des droits de la moitié de l'Humanité. Une violation qui s'est appuyée sur des principes biologiques propres à la femme et non sur ses capacités intellectuelles. 
sa séparation elle part en Hollande et se lance dans le monde des lettres et du journalisme pour pourvoir à ses besoins et à ceux de ses deux filles. D'après l'Abbé Glaire, elle travailla au Lardon et à la Quintescence (626). Par contre, dans Histoire Générale de la Presse Française, Claude Bellanger affirme qu'elle «dirigea de 1714 à 1730 la Quintessence des lettres historiques, critiques, politiques, morales et galantes, une feuille satirique rédigée depuis 1689, par Jean-Maximilien Lucas, disciple de Spinoza» (1: 149). Son journalisme était surtout apologétique.

D'autre part, l'article d'Henriette Goldwyn nous éclaire sur la valeur journalistique des Lettres historiques et galantes de Mme du Noyer, ainsi que sur l'importance de la presse clandestine imprimée en Hollande sous la censure de Louis XIV. De surcroît, Eugène Hatin lui attribue le lancement du quotidien La Spectatrice (Les Gazettes de Hollande 181-183). Aussi, diverses études critiques de ce journal apportent des informations très intéressantes sur ce journalisme féminin du XVIII ${ }^{\mathrm{e}}$ siècle (Bochenek-Franczakowa ; Brétéché ; Junqua ; Boulard-Jouslin ; Revon-Rivière).

En France, il y avait aussi une presse clandestine qui était distribuée et à laquelle des femmes participaient:

Le contrebandier [...] Cabaud de Rambaud [vint] s'établir à Paris en 1724 pour y vivre du commerce des nouvelles. [...] Les nouvelles lui sont expédiées de l'étranger sous le couvert de $\mathrm{M}$. de Caumartin ou de la duchesse d'Estrée. [...] La bande des nouvellistes est secondée par les quatre sœurs Pommier. La plus jeune, Marguerite, est la maîtresse de Tollot. Laînée, Geneviève, une belle fille aux yeux noirs, a la passion des nouvelles et des nouvellistes. (Hallays 1)

D'un côté, nous avons la duchesse d'Estrée qui faisait office de reporter, et de l'autre nous avons quatre sœurs qui collaboraient au «gazetin». En tout cas, pour Alain Hallays, cela est certain pour Geneviève Pommier. Cependant, Bellanger ne leur octroie pas une participation en tant que «journalistes». Pour lui, les sœurs Pommier «servirent aussi bien de muses que de colporteurs» $(1: 178)$.

Marie Anne Doublet (1677-1771), femme de lettres, salonnière et journaliste avant l'heure, osa, avec beaucoup de talent, défier la loi des hommes en réunissant dans son salon, nommé la «Paroisse», philosophes, politiciens, écrivains et artistes de prestige, qui apportaient les nouvelles fraîches, et les 
présentaient aux assistants. La source de ces scoops provenait de divers coins de France, mais «Mme Doublet se réservait la capitale» (Bellanger 1: 180). Les indiscretions de la cour du roi Louis XV qu'elle faisait publiques faillirent la mener au couvent:

Le neveu de Mme Doublet, le duc de Choiseul, la menace de la faire enfermer dans un couvent en 1762 si elle continue de répandre des nouvelles aussi impertinentes que contraires au service du roi [À sa mort], les Mémoires secrets célébrèrent son talent et reconnurent sa hardiesse. (Bellanger 1:184)

Les soixante ans de travail de Marie Anne Doublet, et de ses collaborateurs, furent imprimés sous le titre: Les Mémoires secrets pour servir à l'histoire de la République des lettres (1777) et représentent, au total, trente-six volumes faisant office d'un grand journal au service de l'Histoire.

Au même titre, nous pourrions également citer: Marie-Jeanne LHéritier (1664-1734) qui revendique la véracité (un des leimotiv du journalisme du $\mathrm{XVIII}^{\mathrm{e}}$ siècle) de ses histoires dans Les Caprices du destin, ou Recueil d'histoires sincères (1718) et dont ses œuvres poétiques sont publiées dans le Nouveau Mercure; Marie-Jeanne Phlipon, plus connue sous le nom de Mme Roland (1754-1793), grande figure de la Révolution et des lettres, qui débuta dans le journalisme avec des articles publiés dans le Courrier de Lyon, le Patriote Français et La Nouvelle Minerve (publications posthumes) (Claude Perroud 403-422) ou encore, dans un autre genre, Jeanne-Marie Leprince de Beaumont (1711-1780) qui publia, outre son fameux conte La Belle et la Bête, plusieurs journaux pour la jeunesse ou Marie Anne Barbier (1670-1745) qui reçut, pour une durée de dix ans, le privilège de publier ses Saisons littéraires ou Mélanges de poésies, d'histoire et de critique (Sgard), ainsi que bien d'autres.

En effet, le Dictionnaire des journalistes dirigé par Jean Sgard cite encore neuf autres noms: Louise de Roussillon Tronchin Dubreuil (1690 ?-1763) qui commença à diriger la Gazette d'Amsterdam en 1743 (Hatin, Les Gazettes de Hollande 164); Marie-Claudine de Saint-Aubin (1727-1790), mère de Madame de Genlis qui dessina un projet de journal féminin innovateur sous le titre de Courrier de la Nouveauté qui ne parut jamais. Son idée lui fut dérobée par un certain Mercier de Menneville (Van Dijk 184); la très connue Jeanne Riccoboni (1713-1792), femme auteur, traductrice et dramaturge qui créa également un périodique en forme de «spectateur» intitulé L'Abeille et qui était inséré dans Le Monde (Sgard); Marguerite Pages-Marinier (1725-1786)

Feminismo/s 34, diciembre 2019, pp. 53-77 
dont «son activité journalistique débute avec le lancement des Annonces, affiches et avis divers de Montpellier le 19 mars 1770 (Sgard); Isabelle Van Zuylen de Charrière (1740-1805) qui faisait du journalisme politique et écrivait sur les problèmes qui survenaient en Hollande et en France (Sgard); Adélaïde Dufrenoy (1765-1825) fut directrice et rédactrice du Courrier lyrique et amusant de 1787 à 1789 et rédigea plusieurs articles pour le Petit Magasin des dames, la Gazette de France et L'Abeille. Elle dirigea aussi la Minerve littéraire (Sgard); Justine Souverant, veuve Giroud (?-1798) qui fut aussi imprimeur-libraire de 1767 à 1798 et publia les Affiches, annonces et avis-divers du Dauphiné, un hebdomadaire qui passa d'une feuille à trois (E.M. Petite Revue des Bibliophiles Dauphinois 202); Maria Patoillat, veuve Ceinglen (?-1713) qui fut condamnée à l'exil en 1687 pour avoir publié les Nouvelles Solides et Choisies. En Hollande, elle dirigea la Gazette de Rotterdam (Bots 31-40); Madeleine Fauconnier (? - ?) qui créa L'Annonce des Deuils de Cour et l'Almanach des Deuils (Grimmer).

Entre journalisme pour femmes et journalisme politique, elles ont toutes été spectatrices actives et divulgatrices des événements de leur époque.

\section{3. À l'aube du journalisme féminin: Le Journal des Dames}

Pour compléter et clore ce siècle si riche en productions journalistiques au féminin et si peu connues, nous citerons le premier journal mensuel féminin et féministe avant la lettre: Le Journal des Dames, créé en 1759 par Thorel de Campigneulles, mais dirigé par Françoise Puzin de La Martinière Benoist (1724-1809?), une femme de lettres et journaliste sous l'ancien Régime. Elle rédigea également le Journal en forme de lettres, mêlé de critiques et d'anectodes en 1757 (Sgard) et inséra «quelques pensées détachées dans quelques journaux» (Histoire littéraire des femmes françoises 318).

Outre les rédacteurs, il faut souligner l'apport des rédactrices telles que Madame de Beaumer (1720-1766), Madame de Maisonneuve (17 ?-1774) et Marie-Émilie de Montanclos, née Mayon (1736-1812) qui «publia le Journal des Dames, [et] eut bientôt tout le succès désirable, parce qu'elle l'enrichissait de sa prose légère et de ses vers agréables» (Paban 125). Toutes trois ont voulu s'adresser aux femmes de leur temps et de leur condition. Ce public féminin pouvait aussi bien être cultivé que pieux ou bien encore sans aucune

Feminismo/s 34, diciembre 2019, pp. 53-77 
prétention savante. Chacune y a trouvé de quoi s'instruire ou simplement de quoi se distraire.

Le Journal des Dames fut bien plus qu'un journal féminin. En effet, Évelyne Sullerot a fait une étude quantitative sur les mots qui apparaissaient dans celui-ci, et est arrivée à la conclusion que des thèmes dits «savants», comme la politique et la philosophie étaient de la partie, «ce qui prouve que les femmes cultivées, bien que dépourvues de tout droit civique, s'intéressaient à la politique» (Bellanger 1: 317), ainsi qu'aux préoccupations propres au sexe féminin.

Une des premières et plus vastes études réalisée sur le journalisme féminin du XVIII ${ }^{\mathrm{e}}$ siècle est celle de Suzan van Dijk. Lauteure y visibilise un grand nombre de femmes journalistes, ainsi qu'un certain type de presse, innovatrice pour l'époque, réalisée par et pour les femmes. De plus, elle met en rapport deux domaines très unis: la littérature et le journalisme. En retraçant l'histoire d'une presse féminine, Suzan van Dijk rend compte aussi bien de leur «apport» comme de l'importance de relever ces traces féminines dans les journaux de l'époque, afin d'écrire une nouvelle histoire de la femme et surtout une nouvelle histoire du journalisme.

\section{CONCLUSION}

Définitivement, entre les salons, les lettres qui se métamorphosent en articles et les innovations journalistiques à tendances féminines, les femmes du XVII et XVIII ${ }^{e}$ siècles ont été bien plus que des précieuses ou des instigatrices. En effet, elles ont été des chroniqueuses de talent.

Ainsi, malgré leur présence limitée en nombre et opportunités on s'aperçoit que des femmes ont également participé activement aux fondations d'un journalisme avant l'heure déjà durant ces siècles. Beaucoup d'entre-elles ont consacré leur vie ou une bonne partie de leur vie au journalisme avec plus ou moins de succès, souvent questionné par leurs confrères, et même par un public très conservateur.

Les différentes études fournies depuis quelques années reflètent l'importance de leur contribution dans ce métier pourtant très masculinisé depuis ses racines grammaticales jusqu'à sa pratique. En effet, l'information transmise, qu'elles ont volontiers fait entrecroiser avec l'histoire et la littérature, apporte 
une vision et un discours féminin intéressant non seulement pour l'étude des mœurs et leur condition de femmes sous l'ancien régime, mais aussi pour l'histoire du journalisme, qui devra être complétée.

Femmes journalistes avant l'heure, elles ont indéniablement tracé le chemin aux héritières du XIX et début du XX $\mathrm{XX}^{\mathrm{e}}$ siècles, moment où le journalisme devient une profession à part entière. Dans ce sens, nous poursuivons les recherches sur ces femmes modernes qui ont plongé la tête en avant dans la «mare» de la communication de masse majoritairement phallocentrique.

\section{RÉFÉRENCES BIBLIOGRAPHIQUES}

Adler, Laure. À l'aube du féminisme: Les premières journalistes (1830-1850). Paris : Payot, 1979.

Allain, LAbbé. L’instruction primaire en France avant la Révolution. Paris: Librairie de la Société Bibliographique, 1881. Gallica. 20 juin 2019.

Aronson, Nicole. «Mandane pamphlétaire». Persée 36 (1984): 139-153. 19 juillet 2019. Doi: <https://doi.org/10.3406/caief.1984.1927>.

Baudino, Claudie. Politique de la langue et différence sexuelle: Université Paris 9. Diss. U, Paris 9,1999.

Bellanger, Claude. Histoire Générale de la Presse Française. Vol. 1. Paris: Presses Universitaires de France, 1969.

Bochenek-Franczakowa, Regina. «La Spectatrice, édition critique établie sous la direction d'Alexis Lévrier», Studi Francesi, 176. 2, (2015): 367-368. 19 juillet 2019. <http://journals.openedition.org/studifrancesi/824>.

Bots, Hans. «La Gazette d'Amsterdam entre 1688 et 1699». Les Gazettes européennes de langue française (XVII ${ }^{e}-X V I I I^{e}$ siècles). Ed. C. L. Henri Duranton. Saint-Étienne: Publications de l'Université de Saint-Étienne, 1993. 31-40. Gallica. 20 juillet 2019.

Boulard Jouslin, Claire. «Du Mercure Anglois au Paris Monthly Review: Quelques réflexions sur les phénomènes de transfert et d'influence dans les presses anglaise et française du long XVIIIe siècle». Études Épistémè 26 (2014). 07 juillet 2019. <http://journals.openedition.org/episteme/295>.

Bourgoing de Villefore, Joseph-François. La vie de Madame la Duchesse de Longueville, Première partie. 1738. Web. 11 juillet 2019.

Brétéché, Marion. «Marie-Jeanne LHéritier, Anne-Marguerite Dunoyer et La Spectatrice: généalogie d'une identité auctoriale féminine dans la presse 
francophone (France - Hollande, 1703-1729)». La Spectatrice. Dir. Alexis Levrier. Reims: Épure, 2013. 247-264.

Carrier, Hubert. La Presse de la Fronde (1648-1653). Les Mazarinades: Les hommes du livre. Genève: Librairie Droz, 1991.

Carrier, Hubert. Les Muses Guerrières. Les Mazarinades et la vie littéraire du XVII siècle: courants, genres, culture populaire et savante à l'époque de la Fronde. Paris: Klincksieck, 1996.

Desrosiers-Bonin, Diane. «Le masque du Rieur de la cour de Suzanne de Nervèze». Tangence 77 (2005): 129-142. 16 juillet 2019. Doi: <https://doi. org/10.7202011702ar>.

Desrosiers-Bonin, Diane. «Notice sur Suzanne de Nervèze». SIEFAR, 2005. Web. 16 juillet 2019.

Dictionnaire de l'Académie Française. $6^{\mathrm{e}}$ éd. Vol. 2. Paris, 1835. Web.

Dictionnaire de l'Académie Française. $8^{e}$ éd. Vol. 2. Paris, 1932. Web.

Dictionnaire Universel François \& Latin. Vol. 5. Paris, France: Compagnie des Libraires Associés, 1771. 05 juillet 2019. <https://preview.tinyurl.com/ y6ktaqjb>.

Durand, Marguerite. «Les femmes dans le journalisme». Manuscrits de Marguerite Durand. Tome 3, 1930: 1-11. 20 février 2018. <https://tinyurl.com/ y6aammpz>.

Elmiger, Daniel. «Quelle évolution dans le domaine de la féminisation de la langue? Constitution d'un corpus pour la description diachronique et synchronique des usages». Intersexion. Langues romanes, langues et genre. Éds. Fabienne H. Baider, Daniel Elmiger. München: Lincom, 2012: 97-105.

E.M. Petite Revue des Bibliophiles Dauphinois. Vol. 3. Grenoble: Allier Frères, 1912. Gallica. 15 juillet 2019.

Feyel, Gilles. «Aux origines de l'éthique des journalistes: Théophraste Renaudot et ses premiers discours éditoriaux (1631-1633)». Le Temps des Médias, 2003. 175-189. 15 juillet 2019. <https://preview.tinyurl.com/yyumev5z>.

Furetière, Antoine. Dictionnaire Universel François. Vol. 2. La Haye: A. E. Leers, Éd., 1701. 4 mai 2019. <https://preview.tinyurl.com/y42u23sk>.

Glaire, Abbé. Encyclopédie Catholique. Vol. X. Paris: Parent Desbarres, 1846. Gallica. 15 juillet 2019.

Goldwyn, Henriette. «Journalisme polémique à la fin du XVII ${ }^{e}$ siècle: Le cas de Mme Du Noyer». Femmes savantes, savoirs des femmes: Du crépuscule de la 
Renaissance à L'aube des Lumières. Ed. Colette Nativel. Genève: Librairie Droz S.A., 1999. 247-256.

Grand-Mesnil, Marie-Noëlle. Mazarin, la Fronde et la Presse. Paris: Armand Colin, 1967.

Grimmer, Claude. La femme et le bâtard. Paris: Presses de la Renaissance, 1983. Hallays, André. «En Flânant. Les Nouvellistes à la main». Le Journal des Débats Politiques et Littéraires, 21 janvier 1910: 1-2. Gallica. 15 juillet 2019.

Hatin, Eugène. Histoire Politique et Littéraire de La Presse en France. Vol. 1. Paris: Pourlet-Malassis et de Broise, 1859. Gallica. 16 juillet 2019.

Hatin, Eugène. Les Gazettes de Hollande et la Presse Clandestine. Paris: René Pincebourde, 1865. Gallica. 16 juillet 2019.

Histoire littéraire des femmes françoises, ou lettres Historiques et Critiques. Vol. V. Paris: Lacombe, 1769. Gallica. 23 juillet 2019.

Houdebine-Gravaud, Anne-Marie. "Insécurité linguistique, imaginaire linguistique et féminisation des noms de métiers». Les femmes et la langue. L’insécurité linguistique en question, Lausanne: éd. Singy, Pascal/ Paris: Delachaux et Niestlé, 1998. 155-176.

Houdebine-Gravaud, Anne-Marie. «Femmes/langue/féminisation: Une expérience de politique linguistique en France». Nouvelles Questions Féministes E Questions Féministes 20. 1 (1999): 23-52.

Junqua, Amélie. «Du Spectator à La Spectatrice: les fascinants dédoublements du modèle spectatorial». La Spectatrice (1730). Éd. Alexis Lévrier. Reims: Épure, collection «Héritages critiques», 3, 2013. 265-281.

Kahznadar, Edwige. «Le non-genre académique: Doctrine de la domination masculine en France». Nouvelles Questions Féministes 26.3 (2007): 25-38.

Karl. «À travers Paris. Halles et Marchés». La Semaine des familles. Revue Universelle Illustrée, 19 février, 21 (1859): 328-330. Gallica. 04 juin 2019.

Lachèvre, Frédéric. Claude Le Petit et la Muse de la Cour. Paris: Librairie Ancienne Honoré Champion, 1922. Gallica. 14 juillet 2019.

Lemarchand, Ernest. «Jean Loret et la Muze historique». Bulletin de la Société des amis de Vincennes (1940): 233-251. Gallica. 04 juillet 2019.

Lévêque, Sandrine. De la professionnalisation journalistique à la professionnalisation politique au prisme du genre. Paris: Université Paris 1, 2016.

Loret, Jean. La Muze Historique ou Recueil des Lettres en vers Contenant les Nouvelles du temps, écrites à son Altesse Mademoizelle de Longueville (Livre 1). Paris: B. n. Lyon, mai-décembre 1650. 02 juin 2019. <https://numelyo.bm-lyon.fr>. 
Magne, Émile. «Scarron et la Fronde». Le Mercure de France, 15 avril, LIV.188 (1905): 510-535. Gallica. 26 juillet 2019.

Maira, Daniel. Remontrances, prophéties et confessions de femmes (1575-1650), édition de Jean-Philipe Beaulieu (Compte rendu). Paris: Classique Garnier, 2014. Open Edition. 17 juillet 2019.

Moreau, Claude. Bibliographie des Mazarinades. Vols. 1 et 2. Paris: Jules Renouard et Cie, 1850. Gallica. 24 juillet 2019.

Muriel Andrin et al. (éd.) Femmes et critique(s): Lettes, Arts, Cinéma. Namur: Presses Universitaires de Namur, 2009.

Naudé, Gabriel. Jugement de tout ce qui a esté imprimé contre le Cardinal Mazarin, depuis le sixième janvier, jusques à la Déclaration du premier Avril mil six cent quarante-neuf. 1649. Gallica. 12 juin 2019.

«On dit que». Chantecler, 7 septembre, 46 (1933): 4-6. Gallica. 09 juin 2019.

Paban, Gabrielle (de). Année des Dames. Vol. 2. Paris: Librairie Crevot, 1820. Gallica. 20 juillet 2019.

Perroud, Claude. «Brissot et les Roland. Collaboration des Roland au Patriote Français». La Révolution française. Revue d'Histoire Moderne et Cotemporaine. Dir. A. Aulard. Paris: La Société de l'Histoire de la Révolution, janvier-juin, 34 (1898): 403-422. Gallica. 20 juillet 2019.

Pierrel, Jean-Marie. (dir.) Dictionnaires ATILF/CNRS. Nancy, 2013. <https://www. cnrtl.fr>. 10 juillet 2019.

Pinson, Guillaume. «La femme masculinisée dans la presse mondaine française de la Belle Époque», Clio. Histoire, femmes et sociétés 30 (2009): 211-230. 15 juillet 2019. <http://journals.openedition.org/clio/9471>; DOI: 10.4000/ clio. 9471 .

Plante, Elisabeth. Hybridité et éthos féminin dans La Nouvelle Armide de Suzanne de Nervèze. Montreal, Québec: Université McGill, 2004.

Planté, Christine et Marie-Ėve Thérenty (dir.). Masculin/Féminin dans la presse $d u$ XIX ${ }^{e}$ siècle. Lyon: Presses Universitaires Lyon, 2014.

Rabaut, Jean. Féministes à la «Belle Époque». Paris: Éd. France-Empire, 1985.

Regnault, Louis. «Jean Loret, journaliste avant l'heure», Bulletin municipal de Carentan. 03 juillet 2019. <https://tinyurl.com/yxw82lz8>.

Revon-Rivière, Élise. «De La Spectatrice à Javotte: enquête sur d'improbables promeneuses (avec quelques détours par Jean Jacques Rousseau)». La Spectatrice (1730). Éd. Alexis Lévrier. Reims: Épure, collection «Héritages critiques», 3, 2013. 311-331.

Feminismo/s 34, diciembre 2019, pp. 53-77 
Ribard, Dinah. «Des journalistes à la cour». Open Edition, 2019. 07 juillet 2019. <Doi:10.4000/crcv.17391>.

Roberts, Mary Louise. «Copie subversive: Le journalisme féministe en France à la fin du siècle dernier». Clio Histoire, Femmes et Société, 6 (1997): 230-247. 15 juillet 2019. <http://journals.openedition.org/clio/390>; DOI: 10.4000/ clio.390.

Rosteguy de L'Ancre, Pierre (de). Lincrédulité et Mescréance du sortilège plainement convaincue. Nicolas Buon, 1622. Gallica. 20 juillet 2019.

Rousseau, Jean-Jacques (1846). Oeuvres Complètes. Dialogues. Correspondance. Table. Vol. IV. Paris: Perrotin, Éd., 1846. 15 juillet 2019. <https://tinyurl. com/y5sp6225>.

Schmit, Joseph-Alexandre. «Les Campagnes de Louis XIII en Lorraine écrites de sa propre main». Mémoires de la Société d'Archéologie Lorraine. Vol. X ${ }^{\mathrm{e}}$. Nancy: Imprimerie de A. Lepage, 1868. 346-360. Gallica. 25 juillet 2019.

Sgard, Jean. (dir.). Dictionnaire des Journalistes (1600-1789), 1999. Web.

Sullerot, Évelyne. La presse féminine. Paris: Armand Colin, 1963.

Sullerot, Évelyne. Histoire de la presse féminine en France, des origines à 1848. Paris: Armand Colin, 1966.

van Dijk, Suzan. Traces de femmes: Présence féminine dans le journalisme français du XVIII ${ }^{e}$ siècle. Amsterdam: APA- Holland University Press, 1988.

Veauvy, Christiane et Laura, Pisano. Paroles oubliées: Les femmes et la construction de l'État-Nation en France et en Italie, 1879-1860. Paris: Armand Collin, 1997.

Vergnes, Sophie. «Des discours de la discorde: les femmes, la Fronde et l'écriture de l'histoire». Études Épistémè (2011). 06 juillet 2019. <Doi:10.4000/ episteme.627>.

Vergnes, Sophie. Les Frondeuses. Une révolte au féminin (1643-1661). Ceyzérieu, France: Éditions Champ Vallon, 2013.

Verna, Christine. La Volupté des mots dans Clélie de Mademoiselle de Scudéry. Universidad de Alicante, 2002.

Viennot, Éliane. Non, le masculin ne l'emporte pas sur le féminin! Petite histoire des résistances de la langue française. Donnemarie-Dontilly: Éditions IX ${ }^{\mathrm{e}}, 2014$.

Winn, Colette H. «Susanne de Nervèze». Protestations et revendications féminines. Textes oubliés et inédits sur l'éducation féminine (XVI $-X V I I^{e}$ siècles). Paris: Champion, 2002.

Zedler, Johann H. Grosses vollständiges Universal-Lexicon aller Wissenschafften und Künste. Vol. 23. Halle, 1740. 16 juillet 2019. <https://tinyurl.com/y4b7qufs>. 